\title{
Amélioration de l'offre fourragère par l'association culturale céréale- légumineuse à double usage en zone nord soudanienne du Burkina Faso
}

\author{
M.F. OBULBIGA ${ }^{1 *}$, V. BOUGOUMA ${ }^{2}$ et $\mathrm{H} \mathrm{O} \mathrm{SANON}^{1}$ \\ ${ }^{1}$ Département de Productions Animales. Institut de l'Environnement et de Recherches Agricoles (INERA), \\ 04 B.P. 8647 Ouagadougou 04, Burkina Faso. \\ ${ }^{2}$ Université Polytechnique de Bobo-Dioulasso 01 B.P. 1091 Bobo-Dioulasso, Burkina Faso. \\ *Auteur correspondant ; E-mail : obulferdi@yahoo.fr
}

\section{RESUME}

L'étude a été réalisée dans deux types de terroir du plateau central du Burkina Faso: le centre périurbain de Tenkodogo, situé dans la partie Centre-Est et le terroir agropastoral de Monomtenga, localisé au Centre Sud du pays. Elle a consisté en la mise en place dans chacun des deux sites une association culturale sorgho (variété ICSV 1049) x niébé (variété KVX 745 11P). Ces tests ont été conduits par 74 producteurs volontaires du terroir de Monontenga et 25 agropasteurs péri-urbains de Tenkodogo retenus à partir des critères de choix préalablement élaborés et soumis aux producteurs intéressés par le test dans le village. L'association culturale des deux espèces à double objectif s'est avérée intéressante, eu égard aux productions obtenues bien qu'elles soient modestes. Les rendements de 425,21 $\mathrm{kg} \mathrm{ha}^{-1}$ en grain avec $1233,47 \mathrm{~kg} \mathrm{ha}^{-1}$ en pailles de sorgho et de $235,34 \mathrm{~kg} \mathrm{ha}^{-1}$ en graines avec 891,16 $\mathrm{kg} \mathrm{ha}^{-1}$ en fanes de niébé obtenus par le mode d'association introduit constituent un important appoint alimentaire pour les familles et le bétail des producteurs. Ces résultats obtenus en rendements culturaux montrent que les nouvelles variétés des deux espèces proposées en milieu rural sont adaptées à l'amélioration du disponible fourrager local.

(C) 2015 International Formulae Group. All rights reserved.

Mots clés : Association culturale, sorgho, niébé, production, Burkina Faso.

\section{INTRODUCTION}

Dans les zones tropicales sèches, la saison de pluies correspondant à l'abondance des pâturages naturels demeure très courte. Elle varie de 3 mois (zone sahélienne) à 5 mois (zone soudanienne). Toutefois, en zone agro-climatique nord-soudanienne du Burkina Faso, les pâturages naturels sont essentiellement constitués des graminées annuelles dont les plus fréquentes sont Pennisetum pedicellatum Trin, Schoenefeldia gracilis Kunth, Setaria pallidefusca (Schum) Stapf et Hubb, Andropogon pseudapricus
Stapf, Loudtia togoensis (Pilger) C.E. Hubb, etc. au détriment des graminées pérennes telles que Andropogon gayanus Kunth, Andropogon Ascinodis C.B.Cl, etc. (Yameogo et al., 2013). Cette situation est due aux principales activités de l'homme qui sont la pâture par le bétail, les feux de brousse et la coupe du bois à des fins diverses (Sawadogo, 2009) Durant la saison sèche, s'étendant d'octobre à mai, la difficulté d'alimentation des animaux herbivores est alors au centre des préoccupations des éleveurs. En effet, au cours de celle-ci, les pâturages herbacés, outre 
leur pauvreté en espèces pérennes, sont constitués d'essences sénescentes et des résidus de récolte notamment les pailles de céréales (sorgho, mil, maïs, riz) dont la disponibilité est fonction de la nature et de l'intensité de l'activité agricole dans la zone (CIRAD, 2009). Pendant cette période, les ressources herbacées seules ne suffisent plus à couvrir les besoins qualitatifs des animaux. A ce stade phénologique, elles apportent une ration énergétique à peine satisfaisante et très pauvre en azote (Kagoné, 2000 ; Zampaligré, 2012). Ce problème de disponibilité en quantité et en qualité du fourrage est aggravé par les effets d'autres facteurs non moins importants qui sont la baisse de la pluviométrie, la diminution des surfaces pâturables au profit de l'extension des cultures pour la satisfaction des besoins vivriers d'une population à fort taux de croissance $(3,1 \%)$. L'élevage présent étant de type extensif reste alors très tributaire de ces pâturages naturels au plan alimentaire.

Trois principaux systèmes d'élevage, grands consommateurs de ces ressources alimentaires, sont rencontrés à savoir le système mixte intégré agriculture-élevage pratiqué par les agro-éleveurs sédentaires, le système périurbain caractérisé par une semiintensification orientée principalement vers l'embouche bovine et ovine et enfin, le système agropastoral transhumant dévolu aux pasteurs peuls. Dans le cadre de l'amélioration de l'offre fourragère à ces systèmes, il s'avère donc capital de contribuer au renforcement de l'intégration agriculture-élevage à travers la recherche de voies d'amélioration du disponible et de la qualité du fourrage, tout en tenant compte des besoins vivriers familiaux des producteurs. La présente étude vise donc à améliorer l'offre fourragère par l'association culturale sorgho-niébé à double usage au profit des premiers systèmes évoqués. L'objectif de la présente étude est d'évaluer l'effet de l'association culturale de ces deux composantes sur les rendements en fourrage et grains/graines de chacune en milieux pastoral rural et périurbain.

\section{MATERIEL ET METHODES \\ Sites d'étude}

L'étude est réalisée dans deux types de terroir: le centre périurbain de la ville de Tenkodogo se trouvant à $11^{\circ} 77^{\prime} \mathrm{N}$ de latitude et $0^{\circ} 36^{\prime} \mathrm{W}$ de longitude, et le terroir agropastoral du village de Monomtenga localisé à $12^{\circ} 11^{\prime} \mathrm{N}$ de latitude et $1^{\circ} 18^{\prime} \mathrm{W}$ de longitude. Au plan géomorphologique, ces deux sites d'étude, relevant du plateau central ou bouclier africain sont dominés par les sols ferrugineux tropicaux lessivés indurés qui couvrent $76,6 \%$ du territoire de la province du Boulgou (MAE, 1988). Les autres principaux types de sols présents sous forme d'inclusions sont les sols ferrugineux tropicaux lessivés à concrétions, nodaux ou hydromorphes, les sols bruns eutrophes et peu évolués (PDRDP/BK-BUNASOL, 2004).

Le climat qui y sévit est du type nordsoudanien avec une pluviosité moyenne annuelle comprise entre 600 et $900 \mathrm{~mm}$ (Zoungrana, 1991 ; Dembele, 2010). La saison de pluies dure 4 à 5 mois avec 47 à 64 jours de pluies (juin - octobre). La température annuelle moyenne atteint $27^{\circ} \mathrm{C}$ environ, avec une amplitude thermique annuelle de 5 à $6{ }^{\circ} \mathrm{C}$. La température moyenne des mois les plus chauds (mars à mai) oscille entre 34 et 40,5 ${ }^{\circ} \mathrm{C}$. L'évapotranspiration potentielle (ETP) moyenne journalière des mois de juillet, août et septembre varie de 5,4 à 5,2 mm. Outre le caractère unimodal du régime pluviométrique, il est observé une importante variabilité interannuelle du total pluviométrique, avec une tendance à la baisse.

\section{Matériel végétal}

Le niébé (Vigan unguculata) utilisé est la variété niébé KVX 745-11P. Quant au sorgho (Sorghum almum) utilisé, il s'agit de la variété ICSV 1049. Toutes les deux variétés son issues des collections génétiques de l'Institut de l'Environnement et de Recherches Agricoles (INERA). Il s'agit des variétés à double objectif (fourrage et grains/graines. En effet, les deux variétés ont la faculté de garder leurs tiges et feuilles encore vertes au stade maturité des grains ou graines («stay green »). Ceci permet d'obtenir 
un fourrage de qualité à la récolte contrairement aux autres variétés qui ne possèdent pas ce caractère.

\section{Méthodes}

Le dispositif expérimental a comporté trois (03) traitements suivants sur chaque site:

1. un traitement constitué de la culture pure de sorgho ;

2. un traitement constitué de la culture pure de niébé ;

3. un traitement constitué de deux lignées de sorgho intercalées d'une ligne de niébé ;

4. un traitement constitué des pratiques habituelles paysannes d'association sorghoniébé;

Dans chacun des deux sites, chaque traitement a été conduit par 10 exploitants sur une parcelle de $800 \mathrm{~m}^{2} \quad(40 \times 20 \mathrm{~m})$ par exploitant.

\section{Mise en place et entretien des parcelles}

Les antécédents culturaux des parcelles étaient : l'arachide (44\%), le sorgho (35\%) et le mil ( $8 \%)$, mil, le niébé $(5 \%)$, le mil x niébé (4\%) et le mil $\mathrm{x}$ arachide (4\%). La préparation du sol a consisté en un labour en sol humide à la traction animale. En culture pure, le sorgho et le niébé ont été semés selon les écartements de $80 \mathrm{~cm}$ entre les lignes et $40 \mathrm{~cm}$ entre les poquets sur la ligne, soit une densité de 31626 plants $\mathrm{ha}^{-1}$. En culture associée, les écartements ont été les mêmes avec deux lignes de sorgho pour une ligne de niébé, soit 21084 plants de sorgho ha ${ }^{-1}$ et 10542 plans de niébé $\mathrm{ha}^{-1}$. Les semis du sorgho associé au niébé se son étalés du 24 juin au 18 juillet 2006 conformément aux recommandations techniques correspondantes à chacune des variétés introduites.

Toutes les parcelles ont bénéficié d'au moins deux sarclages durant le cycle végétatif des cultures. Toutefois, durant la campagne, seule la fumure minérale (urée: 46-0-0) a été appliquée à la dose de $50 \mathrm{~kg}$ d'urée ha $^{-1}$ sur les parcelles de sorgho pur au stade montaison et un traitement phytosanitaire à la dose de 1 litre ha ${ }^{-1}$ de Décis sur les parcelles de culture pure de niébé en début de floraison. Le niébé n'a bénéficié d'aucun inoculum.

\section{Paramètres agronomiques mesurés}

La récolte a eu lieu au stade maturité des grains du sorgho et des gousses du niébé. Elle s'est étalée de la deuxième quinzaine d'octobre (niébé) à fin novembre (sorgho). Elle s'est effectuée en prenant soin de laisser une ligne de bordure de chaque côté de la parcelle pour éviter l'effet bordure. Les panicules de sorgho et les gousses de niébé récoltées séparément ont été séchées 15 jours au soleil avant d'être battues et vannées. Les grains ou graines, obtenus ont été pesés et les rendements estimés par ha.

Les fanes et les tiges issues de la récolte de la parcelle utile ont été pesées sur place et un échantillon d'un (01) $\mathrm{kg}$ a été prélevé pour la détermination de la matière sèche (MS) par séchage à l'étuve à $105^{\circ} \mathrm{C}$ pendant $24 \mathrm{~h}$. Le poids de MS obtenu de l'échantillon prélevé a été utilisé pour calculer par extrapolation le rendement MS de fanes et de tiges par ha.

\section{Evaluation de l'association en termes de rendement}

L'évaluation de l'association en termes de rendement a été réalisée à l'aide du Taux de Surface Equivalente (TSE) ou « Land Equivalent Ratio » (LER) qui se définit comme la superficie de terrain en culture pure nécessaire pour produire les rendements atteints par ha de cultures associées. Selon Wiley (1979) cité par N'goran et al. (2011), il se calcule de la manière suivante :

$$
L E R=L A+L B+\cdots+L N=\frac{Y A}{S A}+\frac{Y B}{S B}+\cdots \frac{Y N}{S N}=\sum_{i=1}^{N} \frac{Y N}{S N}
$$

Où :

- YA ....YN = rendement de chaque composante dans l'association

- SA.....SN = rendement de chaque composante en culture pure

Si la valeur de LER est supérieure à 1 , cela signifie que l'association l'emporte sur les cultures pures au point de vue rendement. Dans ce cas précis, l'utilisation du terrain est mieux indiquée pour la pratique de l'association culturale en raison de 
l'insuffisance de l'espace cultivable due à la très forte pression foncière que connaît la zone d'étude.

\section{Analyse statistique}

L'analyse statistique a été faite avec le logiciel StatView version 4.5 en procédant aux analyses de variance et à la comparaison des moyennes au seuil de signification de 5\%.

\section{RESULTATS}

Effet de l'association sur les rendements culturaux de chaque composante Production de grains de sorgho et graines de niébé

Les rendements en grains du sorgho et en graines du niébé sont consignés dans le Tableau 1. Les rendements en grain de sorgho en culture pure obtenus sont $526,64 \mathrm{~kg} \mathrm{ha}^{-1}$ dans le terroir de Monomtenga et $451,82 \mathrm{~kg}$ $\mathrm{ha}^{-1}$ dans le centre périurbain de Tenkodogo, soit une moyenne de 489,23 $\mathrm{kg} \mathrm{ha}^{-1}$. Il n'a pas été observé une différence significative entre les deux sites $(\mathrm{P}<0,05)$. Il en est de même pour les rendements en culture pure de niébé dont la valeur la plus élevée (331, $\mathrm{kg} \mathrm{ha}^{-1}$ ) est obtenue dans le centre périurbain de Tenkodogo. En comparant ces rendements à ceux obtenus en cultures associées, il se dégage que l'association sorgho $\mathrm{x}$ niébé réduit significativement la production de chaque composante $(\mathrm{P}<0,05)$.

Le rendement potentiel de la variété de sorgho utilisée étant 1 à $1,5 \mathrm{t} \mathrm{ha}^{-1}$, la valeur obtenue en culture pure $489,23 \mathrm{~kg} \mathrm{ha}^{-1}$ ne représente que 32,6 à $48,6 \%$ de ce potentiel. Ceci s'expliquerait essentiellement par la non utilisation optimale de la fertilisation minérale, les écarts des dates de semis entre producteurs, les antécédents culturaux et les aléas climatiques. Aussi, il a été constaté une infestation générale des parcelles par l'espèce Striga hermontthica (Delile) Bentham, une adventice dont les dégâts sur les cultures céréalières ne sont plus à démontrer.

Le taux de Surface Equivalente (TSE) ou «Land Equivalency Ratio » (LER) qui correspond à la superficie de terrain en culture pure nécessaire pour produire les rendements atteints par un hectare de culture associée a été calculé. La valeur obtenue est supérieure à 1 pour les deux modes d'association. Le mode d'association: deux lignes de sorgho pour une ligne de niébé qui a été introduit accuse un LER moyen de 1,61 contre 1,60 observé avec les pratiques habituelles du paysan pour l'ensemble des deux sites d'études. Ce qui signifie qu'il y a une symbiose entre les deux variétés associées: sorgho ICSV 1049 et niébé KVX 11P dans les deux modes d'association. Ainsi, le LER de 1,61 obtenu avec le mode cultural introduit indique un avantage en rendement de l'association de $56 \%$ comparativement à la culture pure de chacune des deux composantes. Toutefois, les deux modes ne présentent pas de différence significative au seuil de $5 \%$.

\section{Production de pailles de sorgho et fanes de niébé}

En matière de production des pailles et fanes, il a été observé dans les deux sites d'étude que l'association réduit significativement le rendement de chaque composante par rapport à sa culture pure $(\mathrm{p}<$ 0,05 ). Ainsi, le sorgho en culture pure donne un rendement moyen en pailles de $1333,20 \mathrm{~kg}$ ha $^{-1}$ pour l'ensemble des deux sites d'étude contre $1233,47 \mathrm{~kg} \mathrm{ha}^{-1}$ (Tableau 2) avec le mode d'association introduit, soit une réduction de 7,5\%. Dans le cas du mode d'association traditionnelle, cette réduction de la production atteint $10,7 \%$, mais il n'existe pas de différence significative au seuil de 5\% entre ces pratiques paysannes et le mode d'association introduit.

Le LER moyen le plus élevé $(1,56)$ est observé avec le mode d'association introduit et donne un avantage en rendement de l'association de 56\% comparativement à la culture pure de chacune des deux composantes. Les écarts relativement importants observés entre les minima et les maxima aussi bien pour les rendements (grain et fourrage) témoignent la grande variabilité liée principalement aux facteurs édaphiques, aux pratiques culturales et à la mauvaise répartition spatio-temporelle des pluies. 
M. F. OBULBIGA et al. / Int. J. Biol. Chem. Sci. 9(3): 1431-1439, 2015

Tableau 1 : Rendements en grains du sorgho et gaines du niébé selon le mode cultural.

\begin{tabular}{|c|c|c|c|c|}
\hline Systèmes culturaux & Sites & $\begin{array}{l}\text { Grains sorgho } \\
\left(\mathrm{kg} \mathrm{ha}^{-1}\right)\end{array}$ & $\begin{array}{c}\text { Graines niébé } \\
\left(\mathrm{kg} \mathrm{ha}^{-1}\right)\end{array}$ & LER \\
\hline \multirow[t]{4}{*}{ Culture pure sorgho } & Monomtenga & $\begin{array}{c}526,64 d \\
(460-611) \\
\end{array}$ & - & 1,00 \\
\hline & Tenkodogo & $\begin{array}{c}451,82 \mathrm{~d} \\
(347-638)\end{array}$ & - & 1,00 \\
\hline & Moyenne & $489,23 \mathrm{~d}$ & - & 1,00 \\
\hline & Monomtenga & - & $\begin{array}{c}322,65 \mathrm{~b} \\
(285-380) \\
\end{array}$ & 1,00 \\
\hline \multirow[t]{2}{*}{ Culture pure niébé } & Tenkodogo & - & $\begin{array}{c}331,67 b \\
(347-638)\end{array}$ & 1,00 \\
\hline & Moyenne & - & $327,17 \mathrm{~b}$ & 1,00 \\
\hline \multirow[t]{3}{*}{ Association sorgho x niébé $(2: 1)$} & Monomtenga & $\begin{array}{c}471,62 \mathrm{c} \\
(420-533)\end{array}$ & $\begin{array}{c}212,15 \mathrm{a} \\
(180-240)\end{array}$ & 1,56 \\
\hline & Tenkodogo & $\begin{array}{c}469,40 c \\
(316-474)\end{array}$ & $\begin{array}{c}258,53 \mathrm{a} \\
(196-341)\end{array}$ & 1,67 \\
\hline & Moyenne & $425,21 \mathrm{c}$ & $235,34 \mathrm{a}$ & 1,61 \\
\hline \multirow{3}{*}{$\begin{array}{l}\text { Association sorgho x niébé } \\
\text { pratiques paysannes }\end{array}$} & Monomtenga & $\begin{array}{c}425,32 \mathrm{c} \\
(364-480)\end{array}$ & $\begin{array}{c}221,62 \mathrm{a} \\
(143-300)\end{array}$ & 1,51 \\
\hline & Tenkodogo & $\begin{array}{c}491,18 \mathrm{c} \\
(372-638)\end{array}$ & $\begin{array}{c}208,96 \mathrm{a} \\
(189-238)\end{array}$ & 1,68 \\
\hline & Moyenne & $458,25 \mathrm{c}$ & $215,29 \mathrm{a}$ & 1,60 \\
\hline
\end{tabular}


M. F. OBULBIGA et al. / Int. J. Biol. Chem. Sci. 9(3): 1431-1439, 2015

Tableau 2 : Rendements en pailles du sorgho et fanes du niébé selon le mode cultural.

\begin{tabular}{|c|c|c|c|c|}
\hline Systèmes culturaux & Sites & $\begin{array}{c}\text { Pailles sorgho } \\
\left(\mathrm{kg} \mathrm{ha}^{-1}\right)\end{array}$ & $\begin{array}{c}\text { Fanes niébé } \\
\left(\mathrm{kg} \mathrm{ha}^{-1}\right)\end{array}$ & LER \\
\hline \multirow[t]{3}{*}{ Culture pure sorgho } & Monomtenga & $\begin{array}{c}1536,67 \mathrm{~d} \\
(1140-1860)\end{array}$ & - & 1,00 \\
\hline & Tenkodogo & $\begin{array}{c}1129,74 \mathrm{~d} \\
(1029-1263)\end{array}$ & - & 1,00 \\
\hline & Moyenne & $1333,20 \mathrm{~d}$ & - & 1,00 \\
\hline \multirow[t]{3}{*}{ Culture pure niébé } & Monomtenga & - & $\begin{array}{c}1203,95 \mathrm{~b} \\
(1028-1377)\end{array}$ & 1,00 \\
\hline & Tenkodogo & - & $\begin{array}{c}1320,81 \mathrm{~b} \\
(1044-1752)\end{array}$ & 1,00 \\
\hline & Moyenne & - & $1262,38 \mathrm{~b}$ & 1,00 \\
\hline \multirow[t]{3}{*}{$\begin{array}{l}\text { Association } \\
\text { sorgho x niébé }(2: 1)\end{array}$} & Monomtenga & $\begin{array}{c}1403,92 \mathrm{~b} \\
(1063-1682)\end{array}$ & $\begin{array}{c}808,84 \mathrm{a} \\
(710-918)\end{array}$ & 1,41 \\
\hline & Tenkodogo & $\begin{array}{c}1063,01 \mathrm{~b} \\
(909-1207)\end{array}$ & $\begin{array}{c}973,47 a \\
(736-11271)\end{array}$ & 1,70 \\
\hline & Moyenne & $1233,47 \mathrm{~b}$ & 891,16 a & 1,56 \\
\hline \multirow{3}{*}{$\begin{array}{l}\text { Association } \\
\text { sorgho x niébé } \\
\text { pratiques paysannes }\end{array}$} & Monomtenga & $\begin{array}{c}1233,14 \mathrm{~b} \\
(996-1460)\end{array}$ & $\begin{array}{c}845,22 \mathrm{a} \\
(557-1100)\end{array}$ & 1,38 \\
\hline & Tenkodogo & $\begin{array}{c}1148,42 \mathrm{~b} \\
(981-1323)\end{array}$ & $\begin{array}{c}840,61 \mathrm{a} \\
(668-1007)\end{array}$ & 1,67 \\
\hline & Moyenne & $1190,78 \mathrm{~b}$ & $842,92 \mathrm{a}$ & 1,53 \\
\hline
\end{tabular}

Les moyennes affectées d'une même lettre sur la même ligne pour la comparaison des moyennes entre modes culturaux et sur la même colonne pour la comparaison sorgo et niébé ne diffèrent pas significativement ( $\mathrm{p}<0,05$ ); Les valeurs ( ) indiquent les minima et les maxima. 
DISCUSSION

La production de grains de sorgho et de graines de niébé

L'association culturale des deux espèces à double objectif s'est avérée intéressante, eu égard aux productions modestes obtenues dues principalement aux facteurs de performance observés par Coulibaly et al. (2012) pour les associations maïs/niébé qui sont les dates de labour et de semis, la densité de peuplement et le niveau de fertilité des sols. Les rendements de 425,21 $\mathrm{kg} \mathrm{ha}^{-1}$ en grain de sorgho et de $235,34 \mathrm{~kg} \mathrm{ha}^{-1}$ en graines de niébé obtenus avec le mode d'association introduit constituent un important appoint alimentaire pour les familles des producteurs dans cette zone agro-écologique (plateau central) où les populations sont soumises à des famines saisonnières chroniques (Ouédraogo, 2003). Les rendements habituels observés en système traditionnel dans ladite zone sont de l'ordre de $844 \mathrm{~kg} \mathrm{ha}^{-1}$ de grain de sorgho en culture pure et $156 \mathrm{~kg} \mathrm{ha}^{-1}$ de graines de niébé en association avec le mil (Obulbiga, 1998). En effet, outre son caractère d'améliorateur de la qualité du sol par la fixation de l'azote atmosphérique (Coulibaly et al., 2012), le niébé en légumineuse vivrière joue un rôle de complémentarité avec la céréale dans la diète alimentaire (Garba, 2007). Tout ceci pourrait expliquer la pratique répandue de cette culture dans la région du centre, malgré le fait que les ménages de cette région connaissent l'un des plus faibles taux d'utilisation des engrais minéraux ou la fumure organique qui est estimé à 53,5\% (PNGT, 2011). Egalement, l'association de deux cultures permet aux producteurs de mieux gérer l'espace cultivable (de plus en plus rare) tout en produisant des avantages au niveau du contrôle des mauvaises herbes, de la couverture du sol, de la protection du sol contre l'érosion et de la dispersion des insectes. Zougmoré et al. (2000) montrent dans la même zone climatique que l'association sorgho-niébé permet une réduction de l'érosion du sol de $80 \%$ par rapport à la culture pure de sorgho et de 45 à $55 \%$ par rapport celle de niébé. La valeur moyenne de LER de 1,61 obtenue du mode d'association introduit est similaire à celle de 1,7 observée par Zougmoré et al. (2003) de l'association de variétés améliorées de sorgho (ICSV1049) et de niébé (IAR/1805-4). Enfin, en matière de qualité nutritionnelle, l'introduction du niébé dans le système de culture du sorgho améliore qualitativement la production en augmentant celles des lipides et des protéines sur la même surface. Ceci est confirmé par les travaux de Adjahossou et al. (2009) qui ont montré que l'association culturale arachide-maïs améliore significativement la production de protéines $(24,1 \%$ à $106,2 \%)$ et de lipides $(147,9 \%$ à $386 \%)$.

\section{La production des pailles du sorgho et des fanes du niébé}

Les résultats obtenus en matière de rendement en pailles et fanes ont également montré que l'association culturale des deux espèces à travers des variétés améliorées semble être une des voies de renforcement durable de l'intégration agriculture-élevage par une amélioration quantitative et qualitative $\mathrm{du}$ disponible du fourrager dans le terroir. Comme cela a été constaté au niveau de la production de grains/graines, l'association culturale céréale-légumineuse améliore davantage la teneur en matière azotée du mélange obtenu. Le LER de 1,56 observé avec le mode d'association introduit et donnant un avantage en rendement de l'association de 61 $\%$ sur la culture pure de chacune des deux composantes, demeure un atout certain pour une diffusion de cette technique dans cette zone confrontée de plus en plus au problème de disponibilité de terres cultivables. L'absence de différence significative entre ce mode d'association introduit et le système traditionnel à l'intérieur du même site et entre les deux sites semble confirmer l'efficacité des espèces à double usage dans l'association.

\section{Conclusion}

Les résultats obtenus en rendements culturaux des associations sorgho-niébé montrent que les variétés améliorées des deux espèces sont adaptées à l'amélioration du disponible fourrager pour les systèmes 
d'élevage sédentaire présents: le système mixte intégré agriculture élevage du terroir agropastoral rural et le système périurbain caractérisé par une semi-intensification orientée principalement vers l'embouche bovine et ovine.

Ces résultats montrent également que ces variétés améliorées pourraient permettre de diminuer de façon notable les famines saisonnières chroniques rencontrées les populations de la zone. Il est donc possible d'accroître la production agricole et animale de ladite région par l'intensification du mode cultural : association céréale-légumineuse à double objectif. Pour cela, nous proposons de promouvoir surtout les associations sorghoniébé. Le niébé est une culture importante dans la zone nord-soudanienne du pays, parce qu'il est tolérant à la sécheresse et améliore la qualité du sol par la fixation d'azote, un effet compensateur de la faible utilisation de la fumure minérale ou organique généralement constatée au niveau des ménages de la région du Centre. L'amélioration des systèmes de culture mixte (céréale-légumineuse) constitue donc l'un des moyens pouvant permettre d'assurer la sécurité alimentaire humaine et animale dans cette zone agro-écologique qui constitue l'espace agropastoral essentiel du pays.

\section{REFERENCES}

Adjahossou BS, Adjahossou VN, Adjahossou DF, Edorh P, Sinsin B, Boko M. 2009. Aspects nutritionnels de l'optimisation d'un système de culture associant le maïs et l'arachide au Sud-Bénin. Int. J. Biol. Chem. Sci., 3(5): 1141-1150.

Coulibaly K, Vall E, Autfray P, Sedogo PM. 2012. Performance technico-économique des associtaions maïs/niébé et maïs/mucuna en situation réelle de culture au Burkina Faso : potentialités et contraintes. Tropicultura, 30(3): 147154.

Dembele Y. 2010. Cartographie des Zones Socio-rurales, Burkina Faso. Un Outil d'aide à la Planification pour la Gestion de l'Eau en Agriculture. FAO, 68 p.
Garba AM. 2011. Etude des possibilities d'amélioration des systems de production à base de légumineuses alimentaires (niébé-arachide) dans la zone agroécologique du Fakara, Sud-ouest du Niger). Mémoire Diplôme d'Etudes Spécialisées en Gestion des Ressources Animales et Végétales en Milieux Tropicaux. Filière : Production Végétale. Faculté Universitaire des Sciences Agronomiques de Gembloux, Belgique, $69 \mathrm{p}$.

Kagoné H. 2000. Gestion durable des écosystèmes pâturés en zone nordsoudanienne du Burkina Faso. Thèse de Doctorat. Gembloux, Faculté Universitaire des Sciences Agronomiques, $237 \mathrm{p}$.

N'Goran KE, Kaassin KE, Zohouri GP, N'Gbesso MF, Yoro GR. 2011. Performances agronomiques des associations culturales ignamelégumneuses alimentaires dans le CentreOuest de Côte d'Ivoire. Journal of Applied Biosciences, 43: 2915-2923.

Obulbiga MF. 1998. Caractérisation des ressources agropastorales et des contraintes liées à leur exploitation par les ruminants dans les villages de LuiliNobéré et de Yambassé (Burkina Faso). Mémoire DES. Faculté Universitaire des Sciences Agronomiques de Gembloux, Belgique, $62 \mathrm{p}$.

Obulbiga MF. 2002. Influence de la fumure azotée et du rythme d'exploitation sur la production d'Andropogon gayanus Kunth cultivée en zone nord-soudanienne du Burkina Faso. Mémoire DEA en Gestion intégrée des Ressources Naturelles. Option Nutrition Alimentation. Université Polytechnique de Bobo-Dioulasso, $45 \mathrm{p}$.

Ouédraogo S. 2003. Impact économique des variétés améliorées du niébé sur les revenus des exploitations agricoles du plateau central du Burkina Faso. Tropicultura, 21(4): 294-210.

PDRDP/KB. 2003. Plan de développement du village de Monomtenga (Kombissiri). Rapport final, $56 \mathrm{p}$. 
PDRDP/KB- BUNASOL. 2004. Etude morphopédologique des provinces du Bazèga et du Kadiogo. MRA. Burkina Faso, 72 p.

PNGT2. 2011. Résumé du rapport de l'étude sur: Les conditions et le niveau de vie des ménages ruraux au Burkina Faso dans le context de la decentralisation. Résultats de l'enquête de base PNGT2 2010, 37 p.

Zagré B. 2000. Rapport d'activités de la campagne 1999-2000 en amélioration variétale des oléagineux annuels (arachide, sésame, soja) en zone sud du Burkina Faso. INERA, 32 p.
Zougmoré R, Kamboun F, Outtara K, Guillobez S. 2003. L'association sorghoniébé pour prévenir le ruissellement et l'érosion dans le sahel au Burkina Faso, 5 p.

Zoungrana I. 1991. Recherches sur les aires pâturées du Burkina Faso. Thèse d'Etat, Université de Bordeaux III, 290 p.

Zampaligré N. 2012. The role of ligneous vegetation for livestock nutrition in the sub Sahelian and Sudanian zones of West Africa : Potential effects of climate change. PhD thesis, University of Kassel, Germany, p. 100. 